\title{
Is coracohumeral distance associated with pain-function, and shoulder range of movement, in chronic anterior shoulder pain?
}

\author{
S. Navarro-Ledesma ${ }^{1,2}$, F. Struyf ${ }^{2}$, M. T. Labajos-Manzanares ${ }^{1}$, M. Fernandez-Sanchez ${ }^{3}$ and A. Luque-Suarez ${ }^{1 *}$
}

\begin{abstract}
Background: The aim of this study was twofold: (i) to assess the intrarater reliability of coracohumeral distance; (ii) to investigate the level of association between coracohumeral distance measured by ultrasonography, and pain-disability and shoulder range of movement, in patients suffering from chronic anterior shoulder pain.

Methods: An observational, cross sectional study was carried out. A convenience sample comprised of 87 patients with chronic anterior shoulder pain was assessed from 3 primary care centres. Main outcomes as pain and function were measured through the shoulder pain and disability index. Furthermore, shoulder range of movement-free of pain in shoulder elevation, as well as coracohumeral distance at both 0 and 60 degrees, were collected.

Results: Absence of any correlation was found between coracohumeral distance and shoulder pain and disability index at both 0 and 60 degrees of shoulder elevation. Furthermore, absence of any correlation was found between coracohumeral distance measurements and active shoulder range of movement -free of pain.

Conclusions: There was poor association between coracohumeral distance and shoulder pain and function, as well as with shoulder range of movement, in patients with chronic anterior shoulder pain. Hence, clinicians should consider, not only increasing this space, but also other possibilities in their therapies, when patients with anterior shoulder pain are treated.
\end{abstract}

Trial registration: ACTRN12614000144617. Registered: $1^{\text {st }}$ March 2014.

Keywords: Shoulder pain, Ultrasonography, Diagnosis, Rehabilitation, Chronic pain

\section{Background}

Shoulder pain is one of the most common musculoskeletal conditions in primary care, with a prevalence fluctuating from 6.9 to $26 \%$ for point prevalence, $18.6-31 \%$ for 1 month prevalence, $4.7-46.7 \%$ for 1-year prevalence and $6.7-66.7 \%$ for lifetime prevalence [1] and with 12-month recurrence rates approximately twice the prevalence rates [2]. In the working population, shoulder pain prevalence associated with musculoskeletal disorders is even higher [3].

Anterior shoulder pain has traditionally been underestimated in the assessment of shoulder pain [4]. Although it can occur alone, it usually presents with anterolateral

\footnotetext{
* Correspondence: aluques@uma.es

'Department of Physiotherapy, University of Malaga, Malaga, Spain

Full list of author information is available at the end of the article
}

shoulder pain (labeled as subacromial pain syndrome), sharing similar symptoms [5] and making it difficult to diagnose. The most related cause of anterior shoulder pain is subcoracoid impingement syndrome, defined as the encroachment of the posterolateral coracoid process upon the lesser tuberosity of the humerus [6], causing a compression of soft tissues, such as the subscapularis tendon, glenohumeral joint capsule and subcoracoid bursa, and occasionally the long head of the biceps tendon [7]. Anatomic differences for humerus lesser tuberosity and coracoid process $[6,8]$, as well as anteversion and internal humeral rotation [7], and a history of chronic overuse of persisted flexion, adduction and internal rotation shoulder positions [9], have also been established as possible causes of anterior shoulder pain. 
Diagnosis of anterior shoulder pain has not been widely investigated, but physical examination (cross-arm adduction test) and radiographic features are the most commonly used methods [9]. The coracohumeral interval $(\mathrm{CHI})$ has been measured in previous investigations using the coracohumeral distance (CHD) to determine the severity of anterior shoulder pain $[5,6,10]$, sometimes by means of computed tomography or magnetic resonance imaging (MRI). However, there is a clear lack of standard procedure to quantify it.

Ultrasonography (US) is a non-invasive tool without ionizing effects that permits dynamic evaluation, and is more accessible than those previously described. It has been widely used in the determination of the acromiohumeral distance (AHD) [11, 12]. Two studies have investigated the use of US in the evaluation of CHD $[13,14]$. Oh et al. recently found a good correlation (>0.7) between US and MRI in quantifying CHD, as well as an excellent intra-rater reliability $(>0.90)$ in patients with rotator cuff tear, supporting the use of US in the evaluation of coracohumeral interval. However, there is a lack of a clear measuring process, normative values and reliability data for CHD, measured by US, in patients suffering from anterior shoulder pain. There is also inconclusive evidence on the association of anterior shoulder pain with pain-function and shoulder range of movement (ROM), in patients with chronic anterior shoulder pain. The role of acromiohumeral distance (AHD) as an explanatory factor for symptoms in RC tendinopathy is starting to be questioned [15]. However, the research about whether CHD could play an important role in the explanation of anterior shoulder pain, is unfinished. If a strong relationship between a reduced CHD and high levels of pain existed, decreased shoulder function and limited shoulder ROM would be identified, allowing preventive and therapeutic efforts to be focused on increasing this space. Hence, the aim of this study was twofold: i) to assess the intrarater reliability of CHD at 0 and 60 degrees of scapular elevation measured by US, in patients suffering from chronic anterior shoulder pain; ii) to determine the association between CHD with shoulder pain, function and shoulder-ROM free of pain.

\section{Method}

\section{Procedure}

A convenience sample of 102 patients with unilateral chronic anterior shoulder pain (more than three months), and with clinical symptoms of anterior shoulder pain, was recruited from three different primary care centers. General practitioners (GPs) carried out the recruitment. Then, research assistants assessed participants for eligibility. If participants satisfied the inclusion criteria, then they were studied. Five participants declined to participate, and 10 participants did not meet the inclusion criteria, hence, a sample comprised of 87 participants was assessed. Research assistants collected the informed consent for every participant.

All participants in the study gave their written informed consent. Participants had to meet the following inclusion criteria to be classified as anterior shoulder pain $[9,16,17]$ : i) positive cross-arm test; ii) painful arc of movement during forward flexion and/or internal rotation; (iii) elicitation of tenderness throughout palpation of the coracoid process.

Furthermore, other inclusion criteria had to be met: both men and women aged between 18 and 55 years; no history of significant shoulder trauma, such as fracture or clinically/ultrasonographic-suspected full thickness rotator-cuff tear. Participants were excluded from this study if any of these conditions were presented: (i) recent shoulder dislocation, systemic illnesses such as rheumatoid arthritis, and evidence of adhesive capsulitis, as indicated by passive range of motion loss $>25 \%$ in 2 planes of shoulder motion, and loss $>50 \%$ in passive external rotation; (ii) shoulder pain that was deemed to be originating from any passive and/or neck movement or if there was a neurological impairment, osteoporosis, haemophilia and/or malignancies; (iii) shoulder surgery in the last year, (iv) corticoid injections during the 6 months prior to the study; (v) analgesic-antiinflamatory medication intake during $48 \mathrm{~h}$ prior to the study.

\section{Outcome measures \\ Coracohumeral distance (CHD)}

A diagnostic ultrasound unit, Sonosite M-turbo (GE Healthcare, Wauwatosa, WI) with a dynamic range up to $165 \mathrm{~dB}$, was used. Furthermore, a $6-13-\mathrm{MHz}$ linear transducer with 196 piezoelectric crystals with a specific ultrasound system called "SonoMB" multi-beam imaging", to increase resolution and improve visualisation of physiological and subtle tissue differences, was used to capture images in a grey scale of 256 shades. Ultrasound images were obtained by a single examiner, who was a licensed physiotherapist with advanced training in musculoskeletal ultrasound imaging, and 4-years of experience. Three measurements were taken. An interval of 1 minute was provided between measures, encouraging the patient to move freely. Patients were then repositioned and the second and third set of measurements was successively taken. The ultrasound examiner was blind to all measurements (values were obscured by placing a sticker on the ultrasound screen, meanwhile a research assistant took them and put into a dataset). All the ultrasound measures were expressed in centimeters. CHD was measured at 0 and 60 degrees of active shoulder elevation in the scapular plane, neutral shoulder rotation, with the participant seated in an upright position. 
Patients were seated upright without back support, their feet flat on the ground. To guarantee 0 and 60 degrees shoulder elevation, a hydro-goniometer was placed on the patient's arm [18]. CHD was defined as the shortest linear distance between the coracoid and the adjacent humeral head [9]. The ultrasound transducer was placed over the most anterior aspect of the shoulder, observing the coracoid process and the humeral head on the screen, taking the shortest distance between them. CHD was measured in centimeters, using the calipers on the ultrasound screen (Figs. 1 and 2).

\section{ROM-free of pain at shoulder elevation}

Range of movement (ROM) free of pain at shoulder elevation was taken using the same procedure as followed for CHD ultrasonography measures, excepting a change in the patient's position (stand up position). Three measures were taken separated by an interval of 1 minute, and mean was calculated. ROM was expressed in degrees.

\section{Shoulder pain and disability index (SPADI)}

The Shoulder Pain and Disability Index (SPADI) [19] is a self-administered questionnaire that consists of two dimensions, one for pain and another for functional activities. SPADI total score fluctuates between 0 and 100, with $0=$ best and $100=$ worst. SPADI has shown to have good internal consistency (overall Cronbach's alpha = 0.95 ; for the pain subscale $=0.92$; for the disability subscale $=0.93$ ), as well as the ability to detect change over time [20].

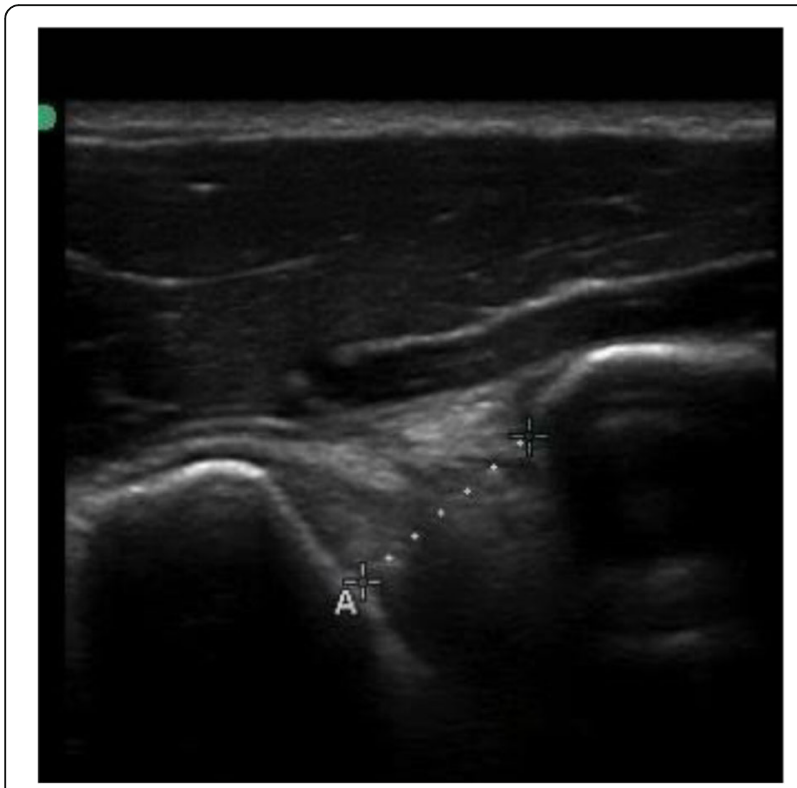

Fig. $1 \mathrm{CHD}$ at 0 degrees of shoulder elevation

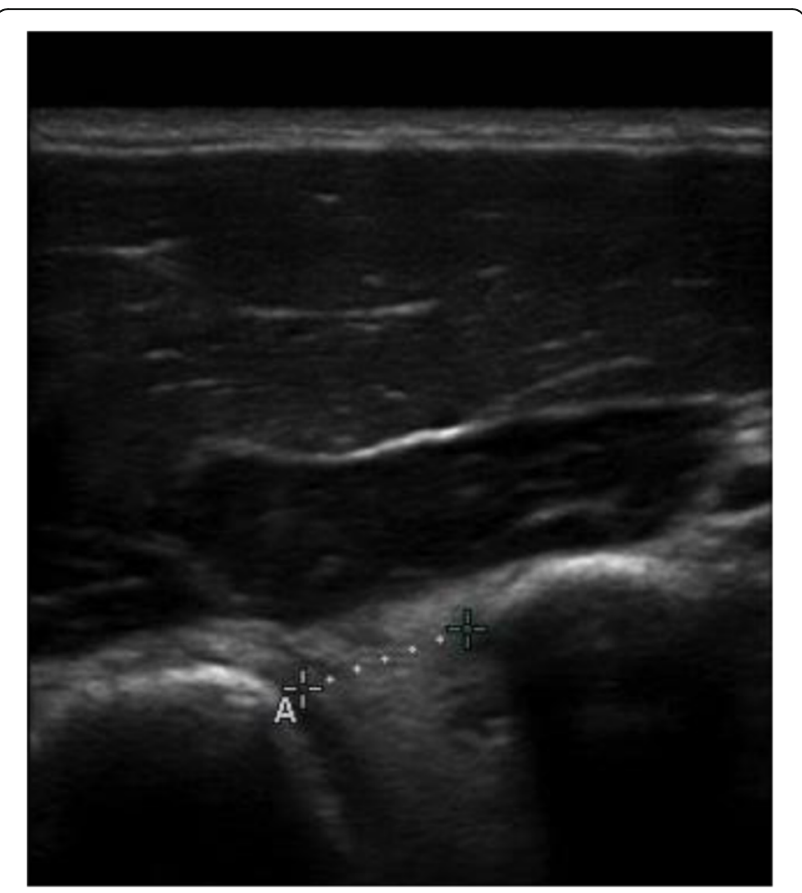

Fig. $2 \mathrm{CHD}$ at 60 degrees of shoulder elevation

\section{Data analysis}

The Statistical Package for the Social Sciences was used for analyzing the collected data (version 23.0 for Mac; SPSS Inc. Chicago, IL). Normality of the variables was visually tested for a Gaussian distribution and additionally tested with a 1-sample Kolmogorov- Smirnov goodness-of-fit test.

For the calculation of reliability of $\mathrm{CHI}$ the model or a 2way mixed consistency intraclass correlation coefficient (ICC) model was used. Hereby a reliability coefficient less than 0.50 was an indication of "poor" reliability; "moderate" between 0.50 and 0.75 , "good" between 0.76 and 0.90 ; and "excellent" over 0.90 [21]. The Standard Error of Measurement (SEM) and the minimal detectable change (MDC) with 95\% confidence bounds (MDC95) were calculated. The MDC has been defined as the minimal amount of change that is required to distinguish a true performance change from a change due to variability in performance or measurement error [22]. To the best of knowledge, there is no studies reporting the MDC in the calculation of intrarater reliability for $\mathrm{CHD}$.

To determine the correlation between $\mathrm{CHD}$ at 0 and 60 degrees with SPADI, and ROM free of pain in scapular plane, Pearson correlation coefficient was calculated for normally distributed data, or Spearman's coefficient in case of absence of normality. Strong correlation was defined as values greater than 0.7 ; between 0.5 and 0.7 correlation was considered moderate; between 0.3 and 0.5 was considered weak correlation [23]. 


\section{Results}

A total sample of 87 patients (71\% women) was assessed. Sample characteristics are presented in Table 1.

Mean values for $\mathrm{CHD}$ at both 0 and 60 degrees are shown in Table 2.

\section{CHD Intra-rater reliability}

Intrarater reliability for $\mathrm{CHD}$ shown excellent values at both 0 and 60 degrees of shoulder elevation (Table 2).

\section{Association between CHD with shoulder pain-function and shoulder-ROM free of pain}

Correlations between CHD, SPADI and shoulder ROM are shown in Table 3.

A poor correlation was found between CHD and SPADI at both 0 and 60 degrees of shoulder elevation. Furthermore, a poor correlation was found between CHD measurements and active ROM-free of pain at shoulder elevation.

\section{Discussion}

The first aim of this study was to determine the intrarater reliability for CHD measured by US in patients suffering from anterior chronic shoulder pain. The results showed an excellent reliability for both 0 and 60 degrees of shoulder elevation. The second aim was to analyze the level of association between CHD and shoulder pain-function as well as shoulder ROM free of pain. Poor associations were noted between the outcomes obtained.

To the best of our knowledge, this is the first and largest study reporting CHD measurements, by means of US, in people suffering from chronic anterior shoulder pain. This study provides results in response to the lack of quality studies in the field of coracohumeral reliability, measured by US. Our findings demonstrated excellent intra-rater reliability for CHD at 0 and 60 degrees (0.98), which are in consonance with Tracy et al. [14] who found an ICC of 0.89 at 0 degrees, in a smaller sample of 19 participants free of shoulder pain. Likewise, Oh et al. [13] achieved intrarater reliability greater than 0.90, in patients with rotator cuff tears. However, the

Table 1 Sample characteristics

\begin{tabular}{ll}
\hline & Mean (SD) \\
\hline Age (years) & $43.9(9.1)$ \\
SPADI (0-100) & $59.7(19.2)$ \\
ROM-free o pain (degrees) & $93.1(33.9)$ \\
Duration of symptoms & \\
& $3-6$ months (26.4\%) \\
& $6-12$ months (13.8\%) \\
& +12 months (59.8\%) \\
\hline
\end{tabular}

Table 2 Intra-rater reliability for CHD at 0 and 60 degrees of shoulder elevation

\begin{tabular}{llllc}
\hline$n(87)$ & mean(SD) & ICC $^{a}$ & SEM & MDC $_{95}$ \\
\hline CHD at 0 degrees & $1.03(0.26)$ & $0.988(0.982-0.992)$ & 0.04 & 0.11 \\
CHD at 60 degrees & $0.94(0.27)$ & $0.989(0.984-0.993)$ & 0.04 & 0.11
\end{tabular}

Intrarater reliability: ICC intraclass correlation coefficient ( ${ }^{a}$ single measure), SEM Standard error of measurement-based on single measure ICC, MDC95 Minimal Detectable Change with $95 \%, \mathrm{Cl}$ based on single measure ICC

position used to measured CHD (cross arm position) in both studies was different in comparison to the present study. The excellent values achieved for CHD measurements are similar to those obtained in similar studies reporting AHD also measured by US, in patients with shoulder pain $[24,25]$. These promising findings are supported by different factors that were considered in the present study in order to improve the quality of the results: (1) the ultrasound examiner was blind to the affected shoulder before measurements were taken; (2) a wash out period of one minute between measurements, allowing patients to move freely between these measurements; (3) no landmarks were used on the skin in an attempt to make every measurement independent with respect to the others; 4) the ultrasound examiner was fully qualified. With respect to the normative values for $\mathrm{CHD}$ in people with shoulder pain, our results showed values of $1.03(0.21) \mathrm{cms}$ at 0 degrees of shoulder elevation, and $0.95(0.25) \mathrm{cms}$ at 60 degrees. Only one study [14] has reported CHD using US, obtaining values of 0.70 (1.4) cms, although CHD was taken in adduction and internal shoulder rotation. This position reduces CHD and so, makes the comparison between findings difficult. MRI has also been used in the assessment of CHD. Specifically, one study has reported values of 0.72 cms [5] in maximal shoulder internal rotation, while with shoulder neutral rotation, values of $1.12(0.33) \mathrm{cms}$ have been found [10], which are in consonance with the results from this paper. Our values were similar in CHD at 0 degrees of shoulder evaluation $(1.03 \pm 0.21 \mathrm{cms})$ to those obtained by $\mathrm{Oh}$ et al. [13] $(1.01 \pm 0.21 \mathrm{cms})$, but in different patient samples (anterior shoulder pain versus full rotator cuff tear).

To the best of our knowledge, this is the first study investigating the relationship of $\mathrm{CHD}$ values, shoulder

Table 3 Correlations between coracohumeral space measured by $\mathrm{CHD}$ at 0 and 60 degrees of shoulder elevation, and SPADI and shoulder ROM free of pain

\begin{tabular}{lcccc}
\hline & SPADI & SEoE $^{\mathrm{a}}$ & ROM & SEoE $^{\mathrm{b}}$ \\
\hline CHD at 0 degrees & $-0.24^{*}$ & 18.61 & $0.23^{*}$ & 32.12 \\
CHD at 60 degrees & -0.15 & 19.16 & 0.19 & 32.10 \\
\hline
\end{tabular}

*: statistically significant $(p<.05)$

aSEoE: Standard error of the estimate (SPADI as dependent variable)

${ }^{\mathrm{b}} \mathrm{SEoE}$ : Standard error of the estimate (ROM as dependent variable) 
pain-function and ROM. It is important to establish the possible association between anterior shoulder pain and CHD measured by US, as well as with active shoulder ROM-free of pain, which could indicate treatments in one direction or another. Our results showed an absence of correlation between CHD and both, SPADI and ROM-free of pain. There are possible underlying mechanisms to explain the low association between CHD, pain and function, and active shoulder ROM-free of pain. Anterior shoulder pain is not a homogenous entity. It seems to appear as a combination of intrinsic factors (for example age, tendon histology and genetics), and extrinsic factors, which are those more closely related to CHD, such as anatomic differences for humerus lesser tuberosity and coracoid process $[6,8]$. Also, anteversion, internal humeral rotation [7], and a history of chronic overuse of persisted flexion, adduction and internal rotation shoulder position [9]. The controversy in regard to the exact pathomechanics and biomechanical causes of shoulder pain is reasonable. This study only shows the level of association between the CHD and the symptoms referred by the patient, not a cause-effect relationship. Since anterior shoulder pain is multifactorial in character, the CHD could only weakly explain the pain perceived and ROM of the patient. Moreover, the chronic character of shoulder pain suffered by the patients included in the present study, could mean the confluence of other possible explanation factors, such as the presence of peripheral-central sensitization, that has been previously reported in shoulder injuries [26]. The present study can only speculate about the real influence of these factors since they were not measured.

There are some limitations that should be taken into consideration. Firstly, inter-rater reliability for ultrasonography measures was not determined; hence results should be taken with caution. Secondly, the difficulty in classifying shoulder pain disorders could mean that heterogeneity is present in the analyzed sample. Hence, previous studies have remarked on the lack of uniformity and reliability in the current diagnostic classification system for shoulder pain [27, 28]. Thirdly, CHD is a two dimensional measurement of a three dimensional space. Therefore, any compromise of this space cannot be completely quantified by the measurement of CHD in isolation. This should be taken into account. Furthermore, the clinical value of CHD must not be outrightly rejected in the clinical assessment of shoulder pain. Finally, due to convenience sample analyzed in this study, results cannot be generalized to other populations.

This study provides promising results regarding the excellent intra-rater reliability of US in the determination of $\mathrm{CHD}$ that quantifies the $\mathrm{CHI}$. Moreover, normative values for $\mathrm{CHD}$ at both 0 and 60 degrees of shoulder elevation in patients with chronic shoulder pain have been identified. However, the real role of the CHD in the explanation of pain severity, alteration of shoulder function and limitation of ROM, in patients with anterior shoulder pain, is not sufficiently clear yet. Hence, future studies should be focused on the determination of its real importance along with other intrinsic and extrinsic factors. This could determine whether it should be considered as a prognostic factor for chronic anterior shoulder pain, and whether it could be an essential factor to guide physical treatments. Furthermore, a standard patient position should be agreed upon when using US as this would make possible comparisons between studies possible.

\section{Conclusions}

In patients with chronic anterior shoulder pain, there is poor association between CHD, and shoulder pain and function, as well as with shoulder ROM-free of pain. Hence, clinicians should consider, not only increasing this space, but also other possibilities in their therapies, when patients with anterior shoulder pain are treated. However, the results should not be generalized to other populations.

\section{Abbreviations \\ AHD: Acromiohumeral distance; CHD: Coracohumeral distance; $\mathrm{CHI}$ : Coracohumeral interval; GP: General practicioner; ICC: Intraclass correlation coefficient; MRI: Magnetic resonance imaging; ROM: Range of movement; SPADI: Shoulder pain and disability index; US: Ultrasonography}

\section{Acknowledgments}

The authors are very grateful to the participants and staff who participated in this project.

Funding

Not applicable.

\section{Availability of data and materials}

Summarized data have been presented in this manuscript. Raw data for this study are located and protected at the University of Malaga, Spain. Sharing of the raw data is not posible, because a secondary analysis is planned.

\section{Authors' contributions}

SNL and ALS made substantial contributions to conception and design acquisition of data, analysis and interpretation of data; were involved in drafting the manuscript and revising it critically for important intellectual content. FS and MTL was involved in drafting the manuscript and revising it critically. MFS supported the data colection and was involved in drafting the manuscript. All authors read and approved the final manuscript.

\section{Competing interests}

The authors declare that they have no competing interests.

Consent for publication

Consent for publication of individual data has been obtained from all the participants of the study.

Ethics approval and consent to participate

This study was approved by the Ethics Committee of Malaga, Spain (PI9/ 012014). This approval was valid for the three primary care centers where this study was carried out. Patients provided consent to participate in the study. 


\section{Publisher's Note}

Springer Nature remains neutral with regard to jurisdictional claims in published maps and institutional affiliations.

\section{Author details}

'Department of Physiotherapy, University of Malaga, Malaga, Spain. ${ }^{2}$ Departament of Rehabilitation Sciences and Physiotherapy, University of Antwerp, Antwerpen, Belgium. ${ }^{3}$ Department of Nursing, Physiotherapy and Medicine, University of Almeria, Almeria, Spain.

Received: 12 January 2017 Accepted: 22 March 2017 Published online: 04 April 2017

\section{References}

1. Luime J, Koes B, Hendriksen I, Burdorf A, Verhagen A, Miedema H, et al. Prevalence and incidence of shoulder pain in the general population; a systematic review. Scand J Rheumatol. 2004;33:73-81.

2. Luime JJ, Koes BW, Miedem HS, Verhaar J a N, Burdorf A. High incidence and recurrence of shoulder and neck pain in nursing home employees was demonstrated during a 2-year follow-up. J Clin Epidemiol. 2005;58:407-13.

3. Roquelaure $Y, \mathrm{Ha}$ C, Leclerc A, Touranchet A, Sauteron M, Melchior M, et al. Epidemiologic surveillance of upper-extremity musculoskeletal disorders in the working population. Arthritis Care Res. 2006;55:765-78.

4. Brunkhorst JP, Giphart JE, LaPrade RF, Millett PJ. Coracohumeral distances and correlation to arm rotation: an in vivo 3-dimensional biplane fluoroscopy study. Orthop J Sport Med. 2013;1:2325967113496059.

5. Misirlioglu M, Aydin A, Yildiz V, Dostbil A, Kilic M, Aydin P. Prevalence of the association of subacromial impingement with subcoracoid impingement and their clinical effects. J Int Med Res. 2012;40:810-5.

6. Gerber C, Terrier F, Ganz R. the Role of the Coracoid Process in the Chronic Impingement Syndrome. J Bone Joint Surg Br. 1985;67.

7. Radas CB, Pieper H-G. The coracoid impingement of the subscapularis tendon: a cadaver study. J Shoulder Elbow Surg. 2004;13:154-9.

8. Giaroli EL, Major NM, Lemley DE, Lee J. Coracohumeral interval imaging in subcoracoid impingement syndrome on MRI. Am J Roentgenol. 2006;186: 242-6.

9. Okoro T, Reddy VRM, Pimpelnarkar A. Coracoid impingement syndrome: a literature review. Curr Rev Musculoskelet Med. 2009;2:51-5.

10. Hekimoglu B, Aydin H, Kizilgoz V, Tatar IG, Ersan O. Quantitative measurement of humero-acromial, humero-coracoid, and coraco-clavicular intervals for the diagnosis of subacromial and subcoracoid impingement of shoulder joint. Clin Imaging. 2013;37:201-10.

11. Desmeules F, Minville L, Riederer B, Côté CH, Frémont P. Acromio-humeral distance variation measured by ultrasonography and its association with the outcome of rehabilitation for shoulder impingement syndrome. Clin J Sport Med. 2004;14:197-205.

12. Hébert $L$, Moffet $H$, Dufour M, Moisan C. Acromiohumeral distance in a seated position in persons with impingement syndrome. J Magn Reson Imaging. 2003;18:72-9.

13. Oh JH, Song BW, Choi JA, Lee GY, Kim SH, Kim DH. Measurement of coracohumeral distance in 3 shoulder positions using dynamic ultrasonography: correlation with subscapularis tear. Arthroscopy. 2016;32(8): $1502-8$

14. Tracy MR, Trella TA, Nazarian LN, Tuohy CJ, Williams GR. Sonography of the coracohumeral interval: a potential technique for diagnosing coracoid impingement. J Ultrasound Med. 2010;29(3):337-41.

15. Lewis J. Bloodletting for pneumonia, prolonged bed rest for low back pain, is subacromial decompression another clinical illusion? Br J Sports Med. 2014;49:280-1.

16. Dines DM, Warren RF, Inglis AE, Pavlov $H$. The coracoid impingement syndrome. J Bone Joint Surg Br. 1990;72:314-6.

17. Russo R, Togo F. The subcoracoid impingement syndrome: clinical, semeiologic and therapeutic considerations. Ital J Orthop Traumatol. 1991; 17:351-8.

18. Hbert LJ, Moffet H, McFadyen BJ, Dionne CE. Scapular behavior in shoulder impingement syndrome. Arch Phys Med Rehabil. 2002;83:60-9.

19. Roach KE, Budiman-Mak E, Songsiridej N, Lertratanakul Y. Development of a shoulder pain and disability index. Arthritis Care Res. 1991;4:143-9.

20. MacDermid JC, Solomon P, Prkachin K. The shoulder pain and disability index demonstrates factor, construct and longitudinal validity. BMC Musculoskelet Disord. 2006;7:12.
21. Portney LG, Watkins MP. Statistical measures of reliability. Found Clin Res Appl Pract. 2000;2:557-86

22. Nair PM, Hornby TG, Behrman AL. Minimal detectable change for spatial and temporal measurements of gait after incomplete spinal cord injury. Top Spinal Cord Inj Rehabil. 2012;18:273-81.

23. Mukaka MM. Statistics corner: a guide to appropriate use of correlation coefficient in medical research. Malawi Med J. 2012;24:69-71.

24. McCreesh KM, Anjum S, Crotty JM, Lewis JS. Ultrasound measures of supraspinatus tendon thickness and acromiohumeral distance in rotator cuff tendinopathy are reliable. J Clin Ultrasound. 2015;0:n/a-n/a.

25. Pijls BG, Kok FP, Penning LIF, Guldemond N a, Arens HJ. Reliability study of the sonographic measurement of the acromiohumeral distance in symptomatic patients. J Clin Ultrasound. 2009;38:128-34

26. Sanchis MN, Lluch E, Nijs J, Struyf F, Kangasperko M. The role of central sensitization in shoulder pain: A systematic literature review. Semin Arthritis Rheum. 2015;44(6):710-6

27. Schellingerhout JM, Verhagen AP, Thomas S, Koes BW. Lack of uniformity in diagnostic labeling of shoulder pain: time for a different approach. Man Ther. 2008;13:478-83.

28. Klintberg IH, Cools AMJ, Holmgren TM, Holzhausen A-CG, Johansson K, Maenhout AG, et al. Consensus for physiotherapy for shoulder pain. Int Orthop. 2015:39:715-20.

\section{Submit your next manuscript to BioMed Central and we will help you at every step:}

- We accept pre-submission inquiries

- Our selector tool helps you to find the most relevant journal

- We provide round the clock customer support

- Convenient online submission

- Thorough peer review

- Inclusion in PubMed and all major indexing services

- Maximum visibility for your research

Submit your manuscript at www.biomedcentral.com/submit
Biomed Central 